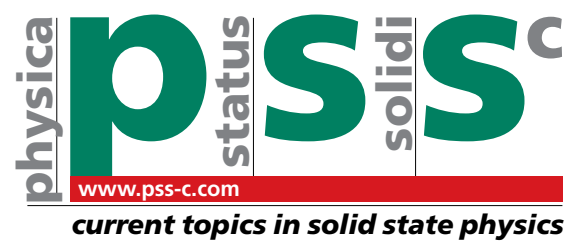

\title{
Using amorphous silicon solar cells to boost the viability of luminescent so- lar concentrators
}

\author{
Daniel J. Farrell ${ }^{\star *}$, Wilfried G. J. H. M. van Sark ${ }^{\star 2,3}$, Steven T. Velthuijsen ${ }^{2}$, and Ruud E. I. Schropp ${ }^{2}$ \\ ${ }^{1}$ Physics Department, Imperial College London, South Kensington campus, SW7 2AZ, London, U.K. \\ ${ }^{2}$ Utrecht University, Faculty of Science, Debye Institute for Nanomaterials Science, Nanophotonics - Physics of Devices, P.O. Box \\ 80000, 3508 TA Utrecht, The Netherlands \\ ${ }^{3}$ Utrecht University, Copernicus Institute for Sustainable Development and Innovation, Science, Technology and Society, \\ Heidelberglaan 2, 3584 CS Utrecht, The Netherlands
}

Received 3 August 2009, revised 4 November 2009, accepted 4 November 2009

Published online 24 February 2010

PACS 84.60.Bk, 84.60.Jt

** e-mail daniel.farrell@imperial.ac.uk (http://www.imperial.ac.uk/qpv)

* Corresponding author: e-mail W.G.J.H.M.vanSark@uu.nl

We have, for the first time, designed and fabricated hydrogenated amorphous silicon solar cells to be used in conjunction with Luminescent Solar Concentrators (LSCs). LSCs are planar plastic sheets doped with organic dyes that absorb solar illumination and down shift the energy to narrowband luminescence which is collected by solar cells attached to the sheet edge.
We fabricated an LSC module with two bonded solar cells and performed characterisation with the cells connected in series and parallel configurations. We find that the LSC module has an optical collection efficiency of $9.5 \%$ and an optimum power conversion efficiency of approaching 1\% when the cells are in a parallel connection.

\section{Introduction}

Luminescent solar concentrators (LSCs) [1] are a possible low cost alternative for widespread deployment of photovoltaic (PV) solar energy converters. These concentrators absorb direct and diffuse light in an organic dye, which can subsequently re-emit, with a slight red-shift. A large fraction of the emission solid angle (75\%) is trapped in the sheet due to total internal reflection and can be collected at the sides of the LSC plate by one or more bonded solar cells, Fig. 1. Of crucial importance is that the organic dyes possess a high fluorescent quantum yield (QY). Currently, organic dyes with near unity QY are mass-produced which span the visible spectrum, however it has not been possible thus far to achieve high QYs for dyes with band gaps larger than $2 \mathrm{eV}(600 \mathrm{~nm})$. This severely limits the spectral range that can be harvested with dyes. One possible approach is to use nanocrystalline absorbers such as quantum dots (QDs) and rods (QRs), in which the absorption edge can be tuned simply by changing the nanostructure size [2]. Although research QDs and QRs have been fabricated with a QY approaching that of dyes this as not yet been replicated in a commercial process.

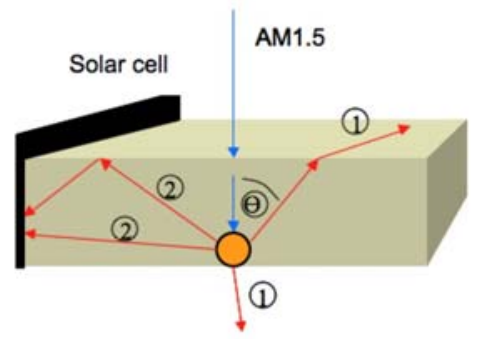

Figure 1 Operation of the LSC: light is absorbed, re-emitted by luminescent dyes and then, depending on emission direction, either escapes the device (rays labels 1 ) or is collected at a bonded PV cell (rays labels 2).

A silicon cell, with a band gap of $1.1 \mathrm{eV}$, while readily available, is therefore not the optimum candidate for a dye based LSC, because the excess energy of the luminescence is lost via thermalisation of the carrier within the cell. Thus, 
conversion efficiencies of 3-4\% have been reported for LSCs with c-Si solar cells. Specially designed III-V cells (such as GaAs and InGaP), with band gaps much closer to the emission energy of the dyes have been recently used and a record efficiency is found of 7.1\% [3]. It remains to be seen whether this can produce an economical photovoltaic product. This is because III-V cells are expensive and typically only used in high concentration (1000x) systems. This is required to reduce the net system cost-perwatt to a competitive level.

It is in this context that we propose to use hydrogenated amorphous silicon cells (a-Si:H) with LSCs as a costeffective alternative to III-V cells. We have designed and fabricated a-Si:H cells for attachment to the side of an LSC plate, that are spectrally matched to the emission of the dyes in the LSC plates (see Fig. 2).

\section{Luminescent concentrator performance}

2.1 Collection efficiency The collection efficiency of a luminescent concentrator is the fraction of the entering photons that are down-shifted, become trapped and are successfully transported to the bonded cells. At each of these stages photon loss mechanisms are introduced: (i) upon absorption there is a chance that non-radiative recombination within the dye molecule occurs, this removes photons from the system, (ii) when trapped, surface imperfections cause scattering which removes photons from waveguide modes, (iii) during transport, non-radiative absorption can occur in the transparent host medium, as well as radiative re-absorption in the dye. For state of the art materials all non-radiative and surface losses are negligible. The main loss mechanism is therefore re-absorption. This is because the following re-emission occurs isotropically, and after many re-absorption-re-emission events trapped photons get redistributed in non-trapped modes and are lost via emission through the top surface.

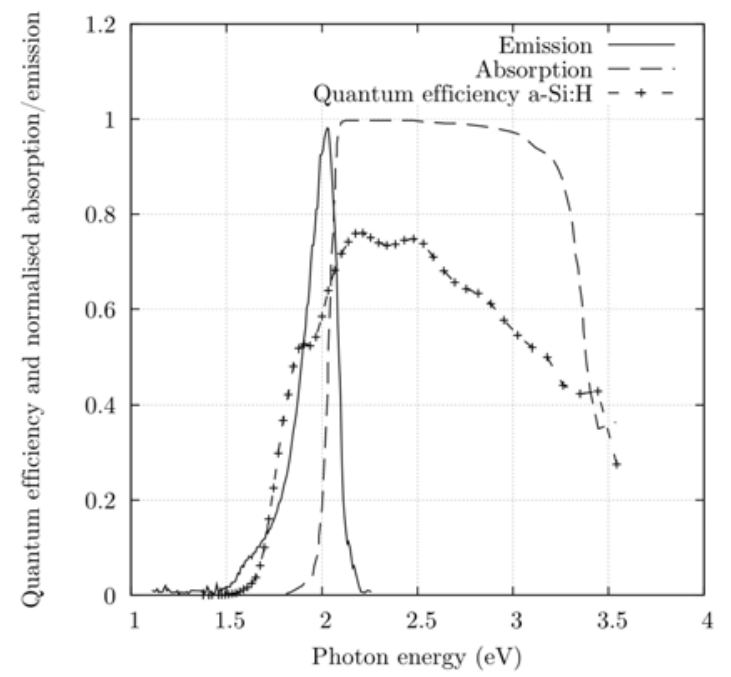

Figure 2 Normalised absorption and emission profile of the LSC sample and quantum efficiency of the a-Si:H cell.
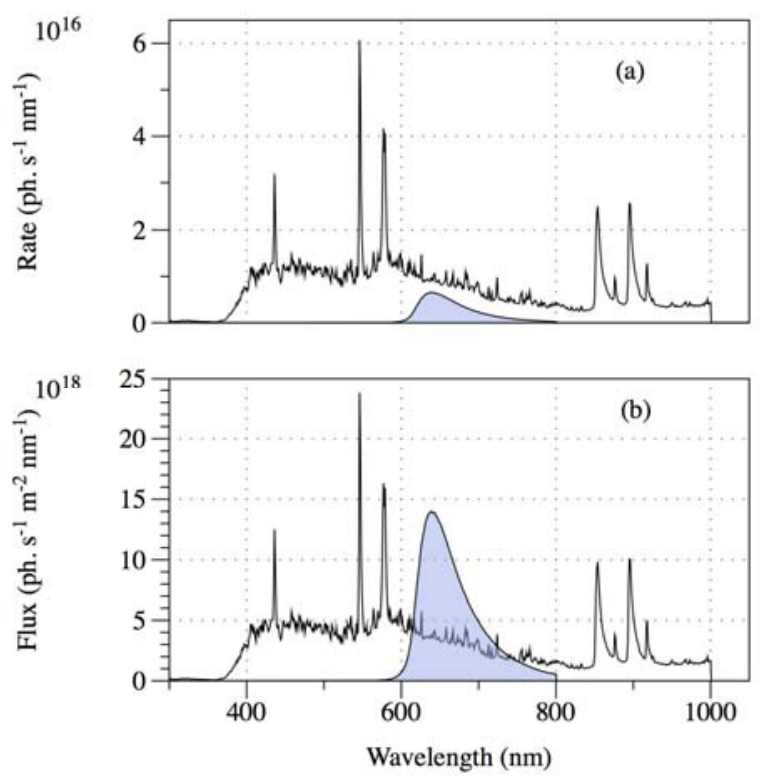

Figure 3 The incident and edge spectra in units of (a) spectral photon rate and (b) spectral photon flux.

Before attaching cells to our LSC sample we first performed the optical characterization discussed below to determine the collection efficiency. The LSC sample was purchased from Lucite (sample code 4T56), cut to dimensions (LxWxD) $5 \times 5 \times 0.5 \mathrm{~cm}^{3}$ and the edges polished. The normalised absorption and emission are shown in Figure 2. The square absorption profile indicates that the plate is doped with multiple luminescent dyes with sequential overlap between the absorption and emission bands. This cascades the absorbed energy to the last dye in the chain and is the reason only red luminescence is observed [4]. To maximize the collection efficiency a PTFE Lambertian back surface reflector with broadband reflectivity $98 \%$ was placed behind the sample during measurements. Any unabsorbed lamp photons or luminescent photons escaping the bottom surface were therefore reflected back into the plate. The reflector was not index matched to the plate, this ensured that total internal reflection is preserved and that the reflector only operates on light that what would otherwise be lost.

A Steuernagel Lichttechnik class B solar simulator with Osram HMI 575W/SE discharge tube was used to produce uniform illumination over the sample surface. The irradiance and photon flux in the plane of the samples top surface and also emitted from the edge was found by measurements with a $2.56 \mathrm{~mm}^{2}$ Siemens BPW34 reference photodiode in combination with an Ocean Optics HR4000 fiber optics spectrometer. The spectrometer was used to find each spectrum in arbitrary units, which was subsequently scaled using reference measurements from the photodiode taken in the same configuration. This yields an incident irradiance of $693 \mathrm{~W} / \mathrm{m}^{2}\left(2.07 \times 10^{21}\right.$ photons $\left./ \mathrm{m}^{2} / \mathrm{s}\right)$ and an escaping luminescent irradiance of $321 \mathrm{~W} / \mathrm{m}^{2}$ 
$\left(1.07 \times 10^{21}\right.$ photons $\left./ \mathrm{m}^{2} / \mathrm{s}\right)$, in the wavelength shown in Fig. 3.

The entering and emitted spectral photon rate (photons $/ \mathrm{s} / \mathrm{nm}$ ) and spectral photon flux (photons $/ \mathrm{m}^{2} / \mathrm{s} / \mathrm{nm}$ ) are shown in Fig. 3a and 3b. There is an important distinction to be made between these figures. The origin of concentration effect at the edges is clearly demonstrated by plotting the spectral photon flux (Fig. 3b), because the emission region of the dye contains much more flux compared to the same region of the lamp spectrum. However, by plotting the spectral photon rate (Fig. 3a), it can be seen that, although brighter, only a fraction of the entering photons are actually emitted from the plate edges. The collection efficiency can be found from the entering and emitted photon flux numbers (quoted above) after multiplication by the areas of the top and edge surface respectively. This procedure is equivalent to taking ratio of the areas under the curves in Fig. 3a. Thus the collection efficiency is found to be $9.5 \%$. The LSC sample has a relatively low collection efficiency. This is because the majority of the incident spectrum (>600 nm) cannot be absorbed in the dyes and also because of emission through the top surface. It is estimated by our Monte-Carlo model that the edge and top surface emission are of similar magnitudes [5].

\section{Device design and fabrication}

3.1 Amorphous silicon solar cells The first design consideration is that the plate edge area $\left(5 \mathrm{x} 0.5 \mathrm{~cm}^{2}\right)$, for optimum collection must also be the active area of the a-Si:H cells. To enable unobstructed photon collection from the LSC a $0.5 \mathrm{~cm}$ wide metal grid contact was located outside of this area. The second design consideration was the spectral location of the a-Si:H spectral response. In this first attempt, we used 'standard' amorphous silicon with a bandgap of $1.75-1.8 \mathrm{eV}$. This yields a cells that is well matched to the luminescence energy of the LSC plate (Fig. 2).

3.2 Luminescent solar concentrators of the cell batches discussed in Section 3.1 the best two cells were selected for bonding to the LSC plate discussed in Section 1.1. The cells were bonded to the LSC edge using Krystalflex PE399 (Huntsman Polyurethane) thermosoftening film following the procedure stated below. A area of PE399 film with dimensions equal to the active area of the cells is cut out and placed on to the top surface of the PV. The cell and film are then heated on a hot plate to $130{ }^{\circ} \mathrm{C}$ for between 2 to 4 minutes. This softens the films at which point the LSC edge is pressed on to the film layer and pressure is applied for a further minute or until a good optical contact has been made. The LSC module (LSC with bonded cells) was then allowed to rest on a heat sink. When cooled, the film made a good optical and mechanical contact between

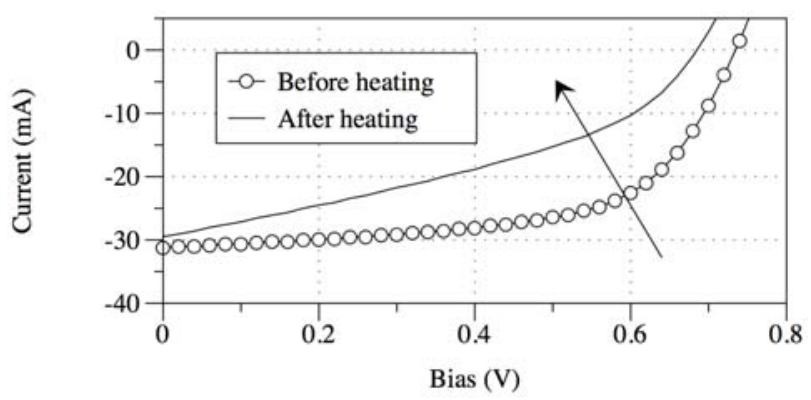

Figure 4 Light IV characteristic of a PV cell before and after 4 minutes heating.

the cell and LSC. The remaining edge area was covered with 3M mirror foil with visible wavelength reflectivity of 95\%. At the back surface the PTFE reflector was placed as in Section 2.1.

To assess the effect the heating and bonding procedure had on the solar cells, the light IV characteristic, of an additional cell was measured before and after 4 minutes heating and pressing. The data in Fig. 4 shows that this procedure degraded the performance by decreasing the shunt resistance. This is probably due to the mechanical stresses involved. Of the two bonded cells only one showed similar degradation in fill factor, while the other showed no degradation from gluing, pressing, and clamping.

\section{Results and discussion}

Light IV measurements were used to characterise the LSC module with illumination provided by the solar simulator. For the best bonded cell this data is compared to a reference measurement of the cell facing the solar simulator (see Fig. 5, only data for one cell is presented due to space limitations). The results show that the bonded cells have a short-circuit current slightly lower (25\% reduction) compared to the value when illuminated directly by the solar simulator. This is caused by a combination of three factors: (i) the collection efficiency of the LSC, (ii) the collection area, and, (iii) the cell response to the luminescent spectrum. Moreover, the collection efficiency of the plate is $10 \%$ but the collection area is only $6 x$ larger than the active edge area. Therefore a lower number of photons enter the cells when bonded than when illuminated directly, even though the collection area has increased. However, from the known collection efficiency and the ratio in areas, one would expect the bonded cells to receive only $60 \%$ (40\% reduction, i.e. $10 \% \times 6$ ) of the photons when bonded, but only $25 \%$ reduction is observed from the IV measurements. The reason for the better than expected performance is because the bonded cells are illuminated by the luminescent spectrum of the dye. This can be converted by the cells at a much higher efficiency than the broadband solar simulator spectrum. The data indicates that under this luminescent spectrum the cells conversion efficiency has almost doubled. 


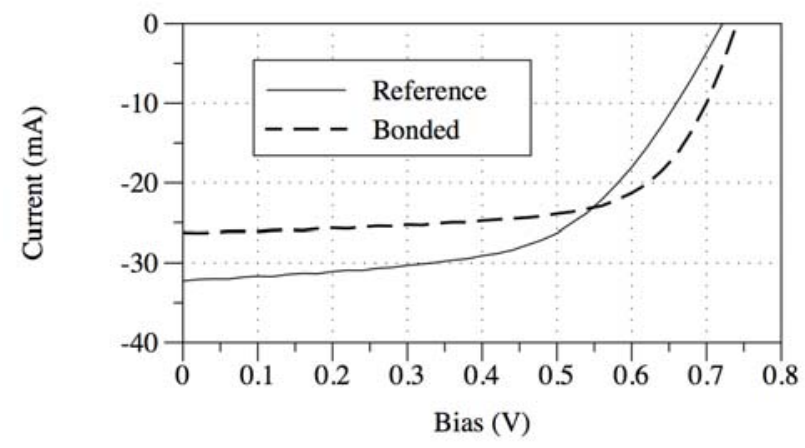

Figure 5 Light IV of the best bonded solar cell under direct illumination by the solar simulator (reference) and when attached to the LSC module (bonded).

There are three possible electrical configurations in which this LSC module can be connected: 4-terminal, series and parallel. The light IV of the LSC module was measured in each configuration. For the 4-terminal connection simple addition of the product of the maximum power point voltages and currents for each cell (of which the 'bonded' curve in Fig. 5 is an example) yields the power out. Thus the LSC module efficiency is defined as the total electrical power out divided by the total entering photonic power (that which is incident on the plate surface). A more likely interconnection scheme is in series or parallel, as this reduces the number of cables required. Figure 6 shows the expected behaviour for series and parallel measurements: when in series the individual IV curves add in (open circuit) voltage and when in parallel they add in (short circuit) current. The degradation of one of the cells unfortunately dominates these measurements, this is seen in the modest current boost when in parallel and the poor FF when in both series and parallel (Fig. 6).

The efficiency and cell performance of each configuration is summarized in Table 1 . The results show that the LSC module had a low power conversion efficiency, about $1 \%$. This is primarily due to relatively low collection efficiency and geometric ratio between the collecting and emitting areas, but also partly due to the degradation of one of the bonded cells. It should be noted that only two of the four edge areas were bonded with cells, so in principle the conversion efficiencies in Table 1 could be almost doubled.

Table 1 Parameters of the LSC module

\begin{tabular}{llllc}
\hline Configuration & $\mathrm{J}_{\mathrm{m}}(\mathrm{mA})$ & $\mathrm{V}_{\mathrm{m}}(\mathrm{V})$ & $\mathrm{FF}$ & Efficiency (\%) \\
\hline Cell \#1 & 12.2 & 0.52 & 0.43 & 0.37 \\
Cell \#2 & 22.2 & 0.58 & 0.66 & 0.74 \\
Series & 14.0 & 0.98 & 0.40 & 0.79 \\
Parallel & 31.6 & 0.5 & 0.48 & 0.91 \\
\hline
\end{tabular}

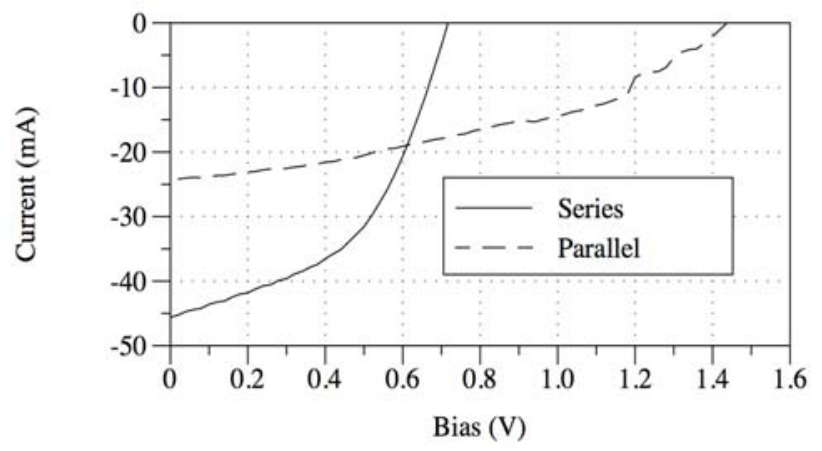

Figure 6 Light IV for the LSC module cells when connected in series (solid line) and in parallel (dashed line).

\section{Conclusion}

We have, for the first time, presented results with aSi:H solar cells and Luminescent Solar Concentrators. The cells were a good spectral matched to the emission of the LSC plate. It was estimated that under the narrowband luminescent spectrum of the LSC, the a-Si:H cells operated at double (around 20\%) their AM1.5 rated efficiency. However, even with this increase in efficiency the low optical collection of the LSC (around 10\%) caused the net power conversion efficiency to be relatively low, approximately $1 \%$.

The low efficiency of the LSC module, although not desirable, should not be considered a barrier to adoption or future development. This is because similar modules to the one described in this paper are useful photovoltaic devices because costs are dramatically reduced. Here we have fused two low cost approaches and so we should not necessarily expect high efficiency. Therefore, the efficiency of the presented LSC module should not be compared to that of the bonded solar cells but instead compared to other low cost approaches such as polymer and organic solar cells, which also operate in and around the $1 \%$ efficiency region.

Acknowledgements The authors would like to thank Karine van der Werf and Caspar van Bommel of the Nanophotonics group at Utrecht University for assistance in designing and fabrication of the amorphous silicon cells and also Markus Führer, Amanda Chatten, Rahul Bose, Ned Ekins-Daukes, Keith Barnham and the other members of the Quantum Photovoltaics Group at Imperial College London for their assistance during device characterisation.

\section{References}

[1] Van Sark et al., Opt. Express 16, 21773-21792 (2008).

[2] Barnham et al. Appl. Phys. Lett. 76, 1197 (2000).

[3] Slooff et al., Phys. Status Solidi RRL 2, 257 (2008).

[4] Richards et al., 19th European Photovoltaic Solar Energy Conference, 7-11 June 2004, Paris, France, 2004, pp. 113.

[5] Farrell, Characterising the Performance of Luminescent Solar Concentrators, University of London, Ph.D Thesis, 2009. 